\title{
Early Growth Stages of Directly Synthesized Large-Area Zeolite Nanosheets
}

\author{
Prashant Kumar, ${ }^{1}$ Mi-Young Jeon, ${ }^{1}$ Michael Tsapatsis, ${ }^{1}$ K. Andre Mkhoyan ${ }^{1}$ \\ ${ }^{1 .}$ Department of Chemical Engineering \& Materials Science, University of Minnesota, Minneapolis, \\ MN.
}

Zeolites are micro- and meso-porous materials, generally synthesized in auto-claves under controlled temperature, pressure and humidity. With the help of organic structure directing agents (SDAs), precisely engineered pore sizes of several zeolite frameworks made of silicon-oxygen tehrahedron, allow for separation of isomeric chemical mixtures containing differently sized molecules like para- and orthoxylene. Top-down synthesis approaches involving exfoliation of a layered precursor material have led to creation of one- and one-and-half unit cell thick zeolite nanosheets [1-3]. However, top-down methods limit the synthesis of large area nanosheets due to the filtration steps involved. This leads to reduced separation performance of the nanosheets as compared to theoretical predictions.

In this study, we have characterized by TEM, a newly developed direct, bottom-up method of fabricating large area MFI-type (3 letter zeolite framework code given by International Zeolite Association [4]) zeolite nanosheets [5]. Tracking the chronological events in growth through ex-situ BF-TEM imaging, diffraction and HAADF-STEM characterization (Figure 1), reveal that the seed particles, (i) are $~ 30 \mathrm{~nm}$ in size at the beginning, (ii) upon further growth elongate preferentially along b-axis of MFI-type unit cell, (iii) trigger a single rotational intergrowth leading to nucleation of a $5 \mathrm{~nm}$ thick, b-oriented MFI nanosheet and (iv) are rapidly encircled by a high-aspect ratio, large area diamond shaped nanosheet. Nucleation of the nanosheet is the key step during this crystal growth modality and TEM investigation of the seed and nanosheet shows that it shares a/b-twin relationship, with the seed being oriented down the a-axis when the sheet is b-oriented. Dark-field TEM reveals that the elongated seed has crystallographic misorientations, while BF-TEM imaging of extended growth periods of the particle compared with Multislice simulations [6] demonstrate the epitaxial thickening of the nanosheets. TEM operating procedures to minimize electron beam damage, Multislice simulations to understand BF-TEM images, crystallographic orientations and epitaxial thickening of the sheet will be discussed in detail [7].

\section{References:}

[1] M. Choi et al, Nature 461, (2009), p. 246.

[2] K. Varoon et al, Science 334, (2011), p. 72.

[3] P. Kumar et al, Nat. Commun. 6, (2015), p. 7128.

[4] http://www.iza-online.org

[5] M.Y. Jeon*, D. Kim*, P. Kumar*, P.S. Lee* et al, Nature (in press, * indicates equal contribution).

[6] E.J. Kirkland in "Advanced Computing in Electron Microscopy", ed. 2, (Springer, New York).

[7] This work was supported as part of the Catalysis Center for Energy Innovation, an Energy Frontier Research Center funded by the U.S. Department of Energy, Office of Science, Basic Energy Sciences under Award DE-SC0001004. 


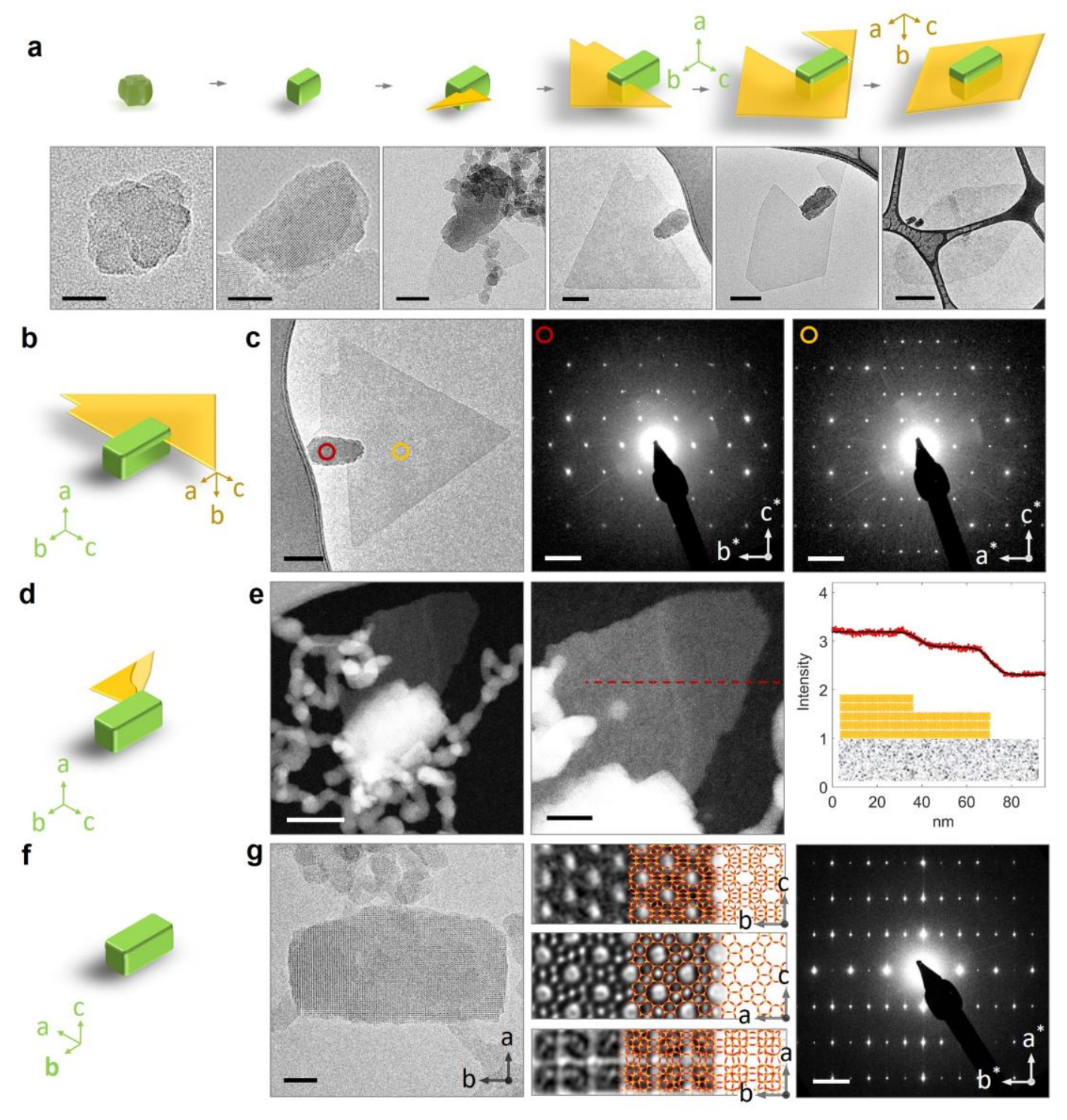

Figure 1. Growth stages of MFI nanosheet from seed. (a) Schematic and the corresponding BF-TEM images representing different stages of growth of MFI nanosheets starting from $\sim 30$-nm size seeds. (b) Schematic showing partial wrap of a b-oriented nanosheet around a-oriented seed. (c) BF-TEM image and corresponding [100] zone axis diffraction pattern from the seed (maroon ring) and [010] zone axis diffraction pattern from the sheet (gold ring). (d) Schematic and (e) HAADF-STEM images of a nanosheet growing out of the seed; Image intensity line-scan along the dotted line in the HAADF-STEM image shows a thickness step in the nanosheet corresponding to a ratio of 5 pentasil chains to 3 pentasil chains from left to right. (f) Schematic showing the preferential growth of a seed along the b-axis. (g) Corresponding TEM image of the seed and the HR-TEM images overlaid with the crystal structure model along [100], [010] and [001] zone axis confirm the MFI-type zeolite structure; Diffraction pattern of the seed taken along [001] zone axis confirms elongation of the seed along b-axis. Scale bars from left to right in (a) are $20 \mathrm{~nm}, 20 \mathrm{~nm}, 50 \mathrm{~nm}, 100 \mathrm{~nm}, 100 \mathrm{~nm}$, and $500 \mathrm{~nm}$, in (c) are $100 \mathrm{~nm}, 1 \mathrm{~nm}^{-1}$, and $1 \mathrm{~nm}^{-1}$, in (e) are $50 \mathrm{~nm}$ and $20 \mathrm{~nm}$, in (g) are $20 \mathrm{~nm}$ and $1 \mathrm{~nm}^{-1}$. 